\title{
Mask similarity impacts short-term consolidation in visual working memory
}

\author{
Lisa Durrance Blalock
}

Published online: 29 May 2013

(C) Psychonomic Society, Inc. 2013

\begin{abstract}
Short-term consolidation is the process by which perceptual representations are stabilized into visual working memory (VWM) representations to prevent interference from subsequent visual input. The present article reports how shortterm consolidation is affected by the similarity of the subsequent visual items (i.e., visual masks) by using a color change detection task. In the task, masks were either similar or dissimilar to the memory stimuli and were displayed at varying intervals following the memory array. The similar masks were made up of the same colored squares as the to-be-remembered stimuli, whereas the dissimilar masks were black-and-white grids. The results showed more interference from similar than from dissimilar masks: Similar masks required more time to consolidate and elicited lower overall performance than did dissimilar masks. These results suggest that a simple overwriting process cannot fully account for the impact of mask type on short-term consolidation performance and that other cognitive mechanisms are involved (e.g., controlled attention).
\end{abstract}

Keywords Visual working memory $\cdot$ Short-term consolidation $\cdot$ Interference $\cdot$ Change detection

Visual working memory (VWM) refers to the set of cognitive processes responsible for encoding, maintaining, manipulating, and retrieving visual information. As VWM receives perceptual input, some mechanism must select some of the visual representations and stabilize them so that they can persist for longer than a few hundred milliseconds and remain active in the face of new visual input (Gegenfurtner \& Sperling, 1993; Luck, 2008). This process of turning a

\section{D. Blalock (ه)}

School of Psychological and Behavioral Sciences, University of West Florida, 11000 University Parkway,

Pensacola, FL 32514, USA

e-mail: lblalock@uwf.edu transient perceptual representation into a durable and stable VWM representation has been termed short-term consolidation (Jolicœur \& Dell'Acqua, 1998; Vogel, Woodman, \& Luck, 2006). The present experiment was designed to examine this process, specifically by examining how the type of interfering visual information affects consolidation.

\section{Short-term consolidation in VWM}

Sperling (1960) showed that, whereas large amounts of visual information can be reported with high accuracy within a few hundred milliseconds following the presentation of a $3 \times 3$ letter matrix, accuracy drops dramatically at longer intervals. Sperling argued that this behavior supported the use of a temporary, high-capacity sensory store following the initial perception of stimuli. However, in order to remember this information for any period beyond a few hundred milliseconds, the use a short-term consolidation process is required, in order to strengthen representations from fleeting sensory memory representations to longer, more stable working memory representations (Gegenfurtner \& Sperling, 1993).

Research has suggested that this consolidation process is very limited in capacity and resource demanding (e.g., Dell'Acqua \& Jolicœur, 2000; Dux \& Marois, 2009; Jolicœur \& Dell'Acqua, 1998). For example, Jolicœur and Dell'Acqua used a dual-task paradigm combining a VWM task and an auditory response task. In this task, a visual array of alphanumeric characters was presented, followed by a low- or highpitched tone. Participants made immediate, speeded responses to the tone and then recalled the characters. Jolicœur and Dell'Acqua found that when the delay between the visual display and the tone was short (i.e., $300-550 \mathrm{~ms}$ ), reaction times to respond to the tone were slow, but reaction times were faster at longer delay intervals. Additionally, this effect was larger when participants had to remember more characters. These results support the use of a consolidation process: That 
is, the visual information needed to be consolidated before participants could respond to the tone, explaining the slower reaction times. Additionally, since more time was required for more items, Jolicœur and Dell'Acqua argued that the consolidation process is limited in capacity, requiring more time as the memory demand increases.

Vogel et al. (2006) examined this idea further by determining the time course of consolidation in VWM. Using a color change detection task, masks consisting of the same colored squares used in the memory array were presented at varying intervals (i.e., stimulus onset asynchronies [SOAs], varying from 117 to 584 $\mathrm{ms}$ ) following the offset of the memory array. The timing of the masks allowed them to examine the time course of consolidation: The masks should only interfere with change detection performance if they were presented while information was still being consolidated into VWM (i.e., at the shorter SOAs). Vogel et al. also assessed whether or not consolidation is resource-limited by varying the set size of the arrays. If consolidation is resourcelimited, more time would be needed to consolidate more items.

They showed that with a set size of one, the mask array did not lead to any drop in performance. However, as the set size increased from two to four, the mask array led to a drop in change detection accuracy at the shorter SOAs, and this effect was larger for larger set sizes. This supports previous research arguing that the consolidation process is limited in capacity, requiring more time as the number to-be-consolidated items increases. Vogel et al. (2006) argued that visual masks overwrite the representations of the memory array unless it has been strengthened into a stable VWM representation.

However, given the categorical similarity of the masks to the memory items, Vogel et al. (2006) noted that the visual masks might compete with the to-be-remembered items for VWM storage. Under this view, the consolidation effects demonstrated previously could be due to poor attentional control in maintaining the memory items at short SOAs (Vogel et al., 2006). If controlled attention is a problem, this would suggest a key role for the central executive component of working memory, which is responsible for controlling attention to maintain goal-relevant information and resist interference from subsequently presented stimuli (Engle, 2002; Engle \& Kane, 2004). Similar masks would make it more difficult for controlled attention to prevent interference, particularly when those items had not been fully consolidated. In order to fully examine the nature of short-term consolidation (i.e., whether its effects are due to overwriting or controlled attention errors), it was necessary to compare masks that were categorically similar and dissimilar to the memory items.

\section{Present experiment}

The present experiment was designed to investigate how mask similarity to target items (in terms of categorical similarity) impacts consolidation using the color change detection task of Vogel et al. (2006). Similar masks were made of the same colors as the to-be-remembered colored squares, whereas the dissimilar masks were black-and-white matrices. If visual masks presented at brief SOAs following the memory array overwrite the to-be-remembered items (as was proposed by Vogel, Woodman, \& Luck, 2006), then both similar and dissimilar masks should impact performance equally (i.e., lower performance at shorter SOAs). Alternatively, if dissimilar masks lead to less interference than do similar masks (i.e., better overall performance and/or less impact of SOA on memory accuracy, this would suggest that other processes are involved in shortterm consolidation (e.g., controlled attention).

\section{Method}

Participants and design

A group of 22 undergraduate psychology students (mean age 21.09 years; 18 females, four males) participated for extra credit in psychology courses. The participants had normal or corrected vision. A 2 (mask type: similar or dissimilar) $\times 4$ (set size: one, two, three, or four items) $\times 5$ (SOA: 117, 234, 350, 467 , or $584 \mathrm{~ms}$ ) within-subjects design was used.

\section{Stimuli}

As in Vogel et al. (2006), seven colored squares were used as the stimuli (RGB values are in parentheses): white $(255,255$, $255)$; red $(227,39,14)$; black $(0,0,0)$; blue $(0,0,225)$; green $(102,250,0)$; violet $(128,21,133)$; and yellow $(225,225,0)$. Each square subtended $0.65^{\circ} \times 0.65^{\circ}$ of visual angle. The items were randomly assigned to positions on an invisible $4 \times 4$ grid
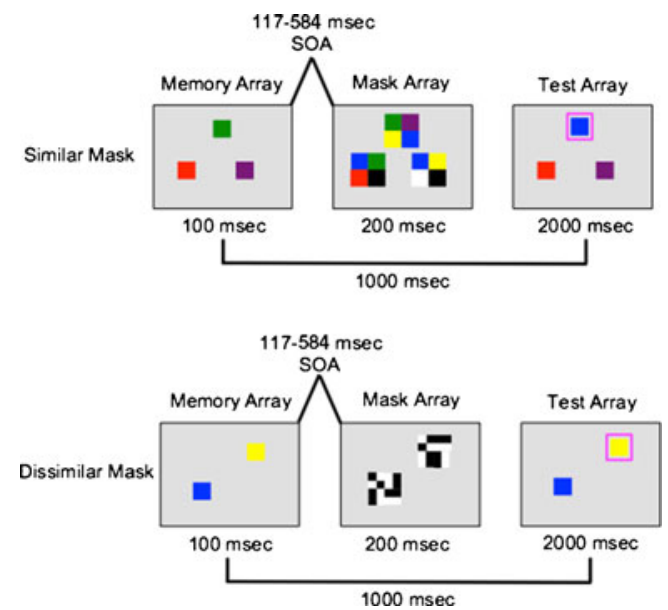

Fig. 1 Example trial procedures for a similar-mask trial, with a set size of three, and a dissimilar-mask trial, with a set size of two. Items are not drawn to scale 
subtending $10.11^{\circ} \times 8.7^{\circ}$ of visual angle, with a minimum item separation of $2.9^{\circ}$. The similar masks consisted of four of the possible colored squares in a $2 \times 2$ checkerboard pattern (Vogel et al., 2006; see Fig. 1, top panels). The colors for each mask were randomly selected with replacement, such that a single color could appear multiple times within the mask array. The dissimilar masks consisted of $4 \times 4$ matrices with half of the cells filled in black and half filled in white (Woodman \& Vogel, 2005; see Fig. 1, bottom panels). Masks were presented at the same locations as the items in the memory array, and the background color was gray $(193,193,193)$ in all conditions.

\section{Procedure}

Each participant was tested in a single session lasting approximately $45 \mathrm{~min}$. Participants completed 400 trials, resulting in ten trials per condition (half with similar and half with dissimilar masks). Trials were presented in a random order for each participant, with the mask type, set size, and SOA conditions intermixed. As is shown in Fig. 1, the memory array was presented for $100 \mathrm{~ms}$, followed by a blank interval until the onset of the mask array. The time of the blank interval varied, with SOAs of 117, 234, 350, 467, and $584 \mathrm{~ms}$ (using the same SOAs as Vogel et al., 2006). A blank screen followed the mask array, the duration of which varied depending on the SOA condition, with a total SOA between the memory and test arrays of $1 \mathrm{~s}$. To avoid comparison errors between the memory and test arrays (Awh, Barton, \& Vogel, 2007), a single item was cued at test with a magenta $(251,0,115)$ box. This cued item was either identical between the memory and test arrays (same) or had been replaced with a different color (different).

\section{Results}

Three participants had overall accuracy that was two standard deviations below the mean and were dropped from the analysis, resulting in 19 participants. For some analyses the sphericity assumption was violated, and the degrees of freedom were thus adjusted using the Huynh-Feldt correction. All pairwise comparisons used the least significant difference adjustment. The results are displayed in Fig. 2.

A 2 (mask type: similar vs. dissimilar) $\times 4$ (set size: one, two, three, or four items) $\times 5$ (SOA: 117, 234, 350, 467, $584 \mathrm{~ms})$ repeated measures analysis of variance (ANOVA) showed significant main effects of mask type $[F(1,18)=13.71, p<.01$, $\left.\eta_{\mathrm{p}}{ }^{2}=.43\right]$, set size $\left[F(2.51,45.22)=49.32, p<.01, \eta_{\mathrm{p}}{ }^{2}=.73\right]$, and SOA $\left[F(4,72)=25.91, p<.01, \eta_{\mathrm{p}}{ }^{2}=.59\right]$, as well as significant Mask Type $\times \operatorname{SOA}[F(2.93,52.77)=6.74, p<.01$, $\left.\eta_{\mathrm{p}}{ }^{2}=.27\right]$ and Mask Type $\times$ Set Size $\times \operatorname{SOA}[F(8.81,158.49)=$ $\left.2.00, p<.05, \eta_{\mathrm{p}}{ }^{2}=.10\right]$ interactions. The Mask Type $\times$ Set

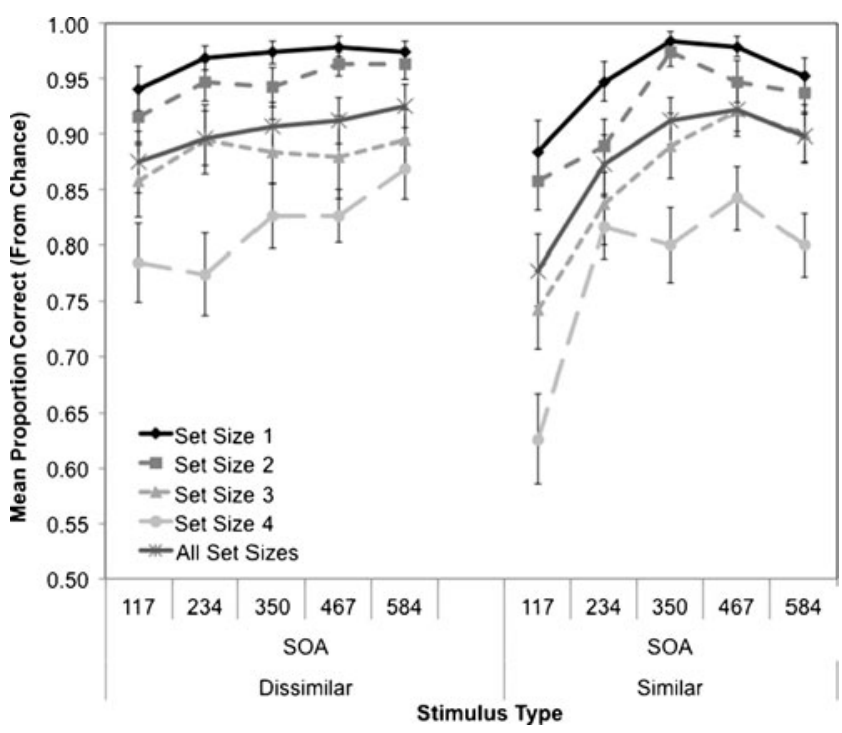

Fig. 2 Mean proportion correct by mask type, set size, and SOA. Error bars represent one standard error of the mean

Size and Set Size $\times$ SOA interactions did not reach significance $\left[\right.$ Mask Type $\times$ Set Size, $F(3,54)=0.50, p=.69, \eta_{\mathrm{p}}{ }^{2}=.03$; Set Size $\times$ SOA, $\left.F(12,216)=0.99, p=.46, \eta_{\mathrm{p}}^{2}=.05\right]$.

Pairwise comparisons on the main effects showed significantly higher accuracy for dissimilar than for similar masks $(p<.01)$, a significant decrease in accuracy with each additional item in the memory array $(p<.01$ for all comparisons), and significant increases in accuracy with each increase in SOA until $350 \mathrm{~ms}$, at which point accuracy remained the same ( $p \mathrm{~s}<.05$ for all comparisons). These results demonstrate that mask similarity impacted performance: Overall, performance was higher with dissimilar than with similar masks, and critically, the three-way interaction was significant, indicating that mask similarity led to changes in consolidation.

To further examine the effects of mask type on consolidation, separate 4 (set size) $\times 5(\mathrm{SOA})$ repeated measures ANOVAs were conducted for the dissimilar and similar masks. For dissimilar masks, significant main effects were found for set size $\left[F(2.25,40.51)=33.19, p<.01, \eta_{\mathrm{p}}{ }^{2}=.65\right]$ and SOA $\left[F(4,72)=3.01, p<.05, \eta_{\mathrm{p}}{ }^{2}=.14\right]$. The Set Size $\times$ SOA interaction did not reach significance $[F(12,216)=0.75, p=.70$, $\left.\eta_{\mathrm{p}}{ }^{2}=.04\right]$. Pairwise comparisons for the main effects showed that performance decreased with increases in set size, with set sizes one and two being marginally significantly different $(p=.055$; $p \mathrm{~s}<.01$ for all other comparisons). Additionally, modest increases in performance emerged with increasing SOA, so that performance was significantly lower at SOAs of 117 and $234 \mathrm{~ms}$ than at the 584-ms SOA ( $p$ s $<.05$ for all comparisons). The lack of a Set Size $\times$ SOA interaction is in contrast with the results of Vogel et al. (2006) and suggests that when dissimilar masks are used, participants are better able to consolidate visual items into VWM, with less interference from intervening masks even at larger set sizes. 
For similar masks, the results showed significant main effects of set size $\left[F(2.70,48.61)=38.40, p<.01, \eta_{\mathrm{p}}{ }^{2}=.68\right]$ and $\operatorname{SOA}\left[F(4,72)=26.66, p<.01, \eta_{\mathrm{p}}{ }^{2}=.60\right]$, as well as a significant Set Size $\times$ SOA interaction $[F(10.33,185.92)=2.15$, $\left.p<.05, \eta_{\mathrm{p}}{ }^{2}=.11\right]$. Pairwise comparisons on the main effects showed that (1) accuracy significantly decreased with each increase in set size, and (2) accuracy increased significantly as SOA increased, with the 117-ms condition having significantly lower accuracy than all other SOA conditions $(p \mathrm{~s}<.01$ for all comparisons); the 234-ms condition having significantly lower accuracy than the 350- and 467-ms conditions ( $p \mathrm{~s}<.01$ ); and no differences between the 350-, 467-, and 584-ms conditions. The significant Set Size $\times$ Delay interaction replicates Vogel et al. (2006), showing that more time is needed to consolidate items into VWM as set size increases. This result contrasts with the dissimilar mask condition, however, demonstrating that similar masks led to greater interference and required more time to consolidate.

\section{Discussion}

The present experiment compared visual masks that were either similar or dissimilar to the memory stimuli in a color change detection task in order to determine how mask similarity affects short-term consolidation of items into VWM. The results showed significantly lower accuracy and greater interference from masking in the similar-mask condition than in the dissimilar-mask condition, especially at larger set sizes. These findings show that a basic overwriting process cannot fully explain the impact of mask similarity on performance, although some evidence of overwriting could still be detected in the dissimilar condition (i.e., a main effect of SOA). The discussion below will focus on what other processes could be involved in short-term consolidation.

\section{Central executive control in short-term consolidation}

First, central executive resources may modulate the consolidation process by keeping the attentional focus on goal-relevant stimuli and blocking intrusions from irrelevant stimuli (Engle, 2002; Engle \& Kane, 2004; Engle, Kane, \& Tuholski, 1999). This becomes harder as the similarity between items increases (Baddeley, 1966a, 1966b; Runquist, 1975). In the present experiment, it is possible that the similar masks made it more difficult for controlled attention to select the to-be-remembered colors from the irrelevant masks, leading to selection errors. This is in line with recent research demonstrating a link between color change detection performance and controlled attention (Blalock, 2012; Fukuda, Vogel, Mayr, \& Awh, 2010). This could also explain why some effect of SOA was apparent with dissimilar masks, since the matrices used did overlap with the black-and-white squares used as memory items.
This result, however, is in contrast to recent research showing no evidence for any impact of stimulus similarity on VWM change detection performance. For example, Lin and Luck (2009) compared VWM performance between similar and dissimilar stimulus conditions (e.g., red, green, and blue vs. three shades of green). Contrary to decades of research in verbal memory, Lin and Luck showed better performance with similar than with dissimilar stimuli. Similarly, Logie, Brockmole, and Vandenbroucke (2009) showed no evidence for long-term learning of stimuli in a change detection task when the stimuli were repeated across trials. Thus, it is possible that similarity impacts on VWM performance and capacity only when similar items are presented soon after the to-beremembered items. Once encoding occurs, those items are then much less susceptible to interference.

This possibility is supported in the present data. When collapsing across set sizes, accuracy was significantly higher in the dissimilar-mask condition only at an SOA of $117 \mathrm{~ms}$ $[t(21)=4.96, p<.01]$, and not at any of the other SOAs, although the difference at $234 \mathrm{~ms}$ was marginally significant [234 ms, $t(21)=2.00, p=.06 ; 350 \mathrm{~ms}, t(21)=0.00, p=1.00$; $467 \mathrm{~ms}, t(21)=0.20, p=.85 ; 584 \mathrm{~ms}, t(21)=1.61, p=.12]$. Thus, the overall advantage for dissimilar masks was driven by masks that were presented very soon following the memory array, at least when the stimuli were simple and highly discriminable. This supports the overall conclusion made here: Although masking can overwrite items as they are encoded into VWM, it also leads to interference in maintaining controlled attention while selecting goal-relevant items for memory.

This conclusion is also in line with recent research by Ueno and colleagues (Ueno, Allen, Baddeley, Hitch, \& Saito, 2011; Ueno, Mate, Allen, Hitch, \& Baddeley, 2011), in which visual suffixes presented after the memory array led to more memory errors, in both recognition and cued recall, when the suffixes shared features (e.g., color or shape) with the target items. Ueno, Allen, et al. (2011; Ueno, Mate, et al., 2011) argued that an attentional, feature-based filter prevents irrelevant stimuli from entering VWM. Items with features similar to those of the target items, however, pass through the filter, at which point they are overwritten. The present results support an attentional filter, but they also demonstrate that it is harder to filter irrelevant items before the to-be-remembered items are fully encoded into VWM.

\section{Perceptual interference}

The controlled-attention explanation argues that the colored masks used in the present experiment led to greater interference because they were in the same category of objects. However, an alternative explanation is that the colored masks interfered with memory more because they were perceptually similar to the memory items. Black-and-white masks, on the other hand, were perceptually dissimilar to the memory items, and thus would lead to lower interference. 
Prior research examining the impact of monochromatic masking on memory for color has been somewhat mixed. In support of the perceptual-interference hypothesis, Hecker and Mapperson (1997) showed greater interference of color flickers for color memory in a cued-recall task than for black-and-white flickers. However, Hecker and Mapperson used a cued-recall task (vs. a recognition task in the present study) and the interfering flickers were shown in the periphery and did not overlap with the memory items. These differences make a direct comparison between their study and the present one difficult, given the differences in recognition versus recall (e.g., Logie et al., 2009; Roediger, 2008) and central versus peripheral vision (e.g., Bouma, 1970; To, Gilchrist, Troscianko, \& Tolhurst, 2011).

In contrast to Hecker and Mapperson (1997), Dent (2009) showed greater interference from monochromatic dynamic visual noise in a color memory task, as compared with a spatiallocation task, using both recognition and cued-recall tasks. The paradigm used by Dent more closely matched the present experiment, in which the dynamic visual noise overlapped with the spatial locations of the memory stimuli. This suggests that monochromatic masking can impair memory for color stimuli. However, given the mixed results in this area, more research will be needed to determine the role of perceptual interference in color memory.

Both the controlled attention and perceptual interference explanations acknowledge that the short-term consolidation mechanism is likely more complex than a basic overwriting process. Indeed, the two views are not mutually exclusive: It is likely that both top-down (controlled attention) and bottom-up (perceptual interference) processes are involved with encoding and stabilizing VWM representations (e.g., Woodman, Vecera, $\&$ Luck, 2003). The backward-masking task provides a useful paradigm for examining the role of each of these processes in future research.

Verbal strategies in color change detection

One limitation of the present study was the lack of an articulatory suppression task to prevent verbal recoding of the stimuli. If participants were verbally recoding the stimuli, similar masks may have led to greater interference in verbal working memory versus VWM. However, prior research indicates adding a verbal suppression task does not impact change detection performance (e.g., Hollingworth, 2003; Luck \& Vogel, 1997), suggesting that participants do not use verbal strategies in change detection tasks. Regardless, to rule out any possible verbal strategies in the present study, a small sample of participants $(N=7)$ completed the experiment while repeating "the" during each trial. With this concurrent suppression task, a significant main effect of mask type still emerged (dissimilar masks $>$ similar masks) $[F(1,6)=9.64$, $\left.p<.05, \eta_{\mathrm{p}}{ }^{2}=.62\right]$, as well as a significant Mask Type $\times$ Set
Size $\times$ SOA interaction $\left[F(12,72)=2.47, p<.01, \eta_{\mathrm{p}}{ }^{2}=.29\right]$, with no Set Size $\times$ SOA interaction for dissimilar masks. This suggests that the greater interference demonstrated for similar masks in the present experiment was due to VWM interference and not to interference in the use of verbal strategies.

\section{Summary}

The present study demonstrates that short-term consolidation is a complex process that involves attentional resources (either bottom-up perceptual or top-down controlled attention processes), which can facilitate very early encoding processes in VWM. From a broader perspective, the present results indicate that perception, VWM, and the central executive, although frequently studied in isolation, are highly interactive, even a few hundred milliseconds following visual input.

Author Note The author would like to thank the following individuals for their help in collecting the data: Tiffany Alviola, Matthew Castagna, Rachael Lagozzino, Jaclynn Lawhon, Raquita Peasant, Christina Sledge, and Cynthia Wallace.

\section{References}

Awh, E., Barton, B., \& Vogel, E. K. (2007). Visual working memory represents a fixed number of items regardless of complexity. Psychological Science, 18, 622-628. doi:10.1111/j.1467-9280.2007. 01949.x

Baddeley, A. D. (1966a). The influence of acoustic and semantic similarity on long-term memory for word sequences. Quarterly Journal of Experimental Psychology, 18, 302-309. doi:10.1080/ 14640746608400047

Baddeley, A. D. (1966b). Short-term memory for word sequences as a function of acoustic, semantic and formal similarity. Quarterly Journal of Experimental Psychology, 18, 362-365. doi:10.1080/ 14640746608400055

Blalock, L. (2012, March). Does fluid intelligence predict stabilization of visual working memory representations? Presented at the Southeastern Psychological Association, New Orleans, LA.

Bouma, H. (1970). Interaction effects in parafoveal letter recognition. Nature, 226, 177-178.

Dell'Acqua, R., \& Jolicœur, P. (2000). Visual encoding of patterns is subject to dual-task interference. Memory \& Cognition, 28, 184 191. doi:10.3758/BF03213798

Dent, K. (2009). Dynamic visual noise affects visual short-term memory for surface color, but not spatial location. Experimental Psychology, 57, 17-26. doi:10.1027/1618-3169/a000003

Dux, P. E., \& Marois, R. (2009). The attentional blink: A review of data and theory. Attention, Perception, \& Psychophysics, 71, 1683-1700. doi:10.3758/APP.71.8.1683

Engle, R. W. (2002). Working memory capacity as executive attention. Current Directions in Psychological Science, 11, 19-23. doi:10.1111/ 1467-8721.00160

Engle, R. W., \& Kane, M. J. (2004). Executive attention, working memory capacity, and a two-factor theory of cognitive control. 
In B. H. Ross (Ed.), The psychology of learning and motivation (Vol. 44, pp. 145-199). San Diego, CA: Elsevier Academic Press.

Engle, R. W., Kane, M. J., \& Tuholski, S. W. (1999). Individual differences in working memory capacity and what they tell us about controlled attention, general fluid intelligence, and functions of the prefrontal cortex. In A. Miyake \& P. Shah (Eds.), Models of working memory: Mechanisms of active maintenance and executive control (pp. 102-134). Cambridge, UK: Cambridge University Press.

Fukuda, K., Vogel, E., Mayr, U., \& Awh, E. (2010). Quantity, not quality: The relationship between fluid intelligence and working memory capacity. Psychonomic Bulletin \& Review, 17, 673-679. doi:10.3758/17.5.673

Gegenfurtner, K. R., \& Sperling, G. (1993). Information transfer in iconic memory experiments. Journal of Experimental Psychology. Human Perception and Performance, 19, 845-866. doi:10.1037/0096-1523. 19.4.845

Hecker, R., \& Mapperson, B. (1997). Dissociation of visual and spatial processing in working memory. Neuropsychologia, 35, 599-603.

Hollingworth, A. (2003). Failures of retrieval and comparison constrain change detection in natural scenes. Journal of Experimental Psychology. Human Perception and Performance, 29, 388-403. doi:10.1037/0096-1523.29.2.388

Jolicœur, P., \& Dell'Acqua, R. (1998). The demonstration of short-term consolidation. Cognitive Psychology, 36, 138-202. doi:10.1006/cogp. 1998.0684

Lin, P.-H., \& Luck, S. J. (2009). The influence of similarity on visual working memory representations. Visual Cognition, 17, 356-372. doi:10.1080/13506280701766313

Logie, R. H., Brockmole, J. R., \& Vandenbroucke, A. R. E. (2009). Bound feature combinations in visual short-term memory are fragile but influence long-term learning. Visual Cognition, 17, 160-179. doi: $10.1080 / 13506280802228411$
Luck, S. J. (2008). Visual short-term memory. In S. J. Luck \& A. Hollingworth (Eds.), Visual memory (pp. 43-85). New York, NY: Oxford University Press.

Luck, S. J., \& Vogel, E. K. (1997). The capacity of visual working memory for features and conjunctions. Nature, 390, 279-281. doi: $10.1038 / 36846$

Roediger, H. L., III. (2008). Relativity of remembering: Why the laws of memory vanished. Annual Review of Psychology, 59, 225-254. doi:10.1146/annurev.psych.57.102904.190139

Runquist, W. N. (1975). Interference among memory traces. Memory \& Cognition, 3, 143-159.

Sperling, G. (1960). The information available in brief visual presentation. Psychological Monographs: General and Applied, 74, 1-29.

To, M. P. S., Gilchrist, I. D., Troscianko, T., \& Tolhurst, D. J. (2011). Discrimination of natural scenes in central and peripheral vision. Vision Research, 51, 1686-1698. doi:10.1016/j.visres.2011.05.010

Ueno, T., Allen, R. J., Baddeley, A. D., Hitch, G. J., \& Saito, S. (2011a). Disruption of visual feature binding in working memory. Memory \& Cognition, 39, 12-23. doi:10.3758/s13421-010-0013-8

Ueno, T., Mate, J., Allen, R. J., Hitch, G. J., \& Baddeley, A. D. (2011b). What goes through the gate? Exploring interference with visual feature binding. Neuropsychologia, 49, 1597-1604. doi:10.1016/ j.neuropsychologia.2010.11.030

Vogel, E. K., Woodman, G. F., \& Luck, S. J. (2006). The time course of consolidation in visual working memory. Journal of Experimental Psychology. Human Perception and Performance, 32, 1436 1451. doi:10.1037/0096-1523.32.6.1436

Woodman, G. F., Vecera, S. P., \& Luck, S. J. (2003). Perceptual organization influences visual working memory. Psychonomic Bulletin \& Review, 10, 80-87. doi:10.3758/BF03196470

Woodman, G. F., \& Vogel, E. K. (2005). Fractionating working memory: Consolidation and maintenance are independent processes. Psychological Science, 16, 106-113. doi:10.1111/j.09567976.2005.00790.x 